\title{
Pelvic Osteosarcoma: Resection and Reconstruction Using A Customised Plate
}

\author{
Mohamed Ali Khalifa ${ }^{1}$, Zeineb Alaya ${ }^{2 *}$, Lassaad Hassini ${ }^{1}$, Mehdi Jedidi $^{1}$, Adnen Siala ${ }^{1}$, Walid Osman ${ }^{1}$, \\ Karim Bouattour ${ }^{1}$ \\ ${ }^{1}$ Department of Orthopaedics, Sahloul Hospital, Faculty of Medicine of Sousse, Tunisia \\ ${ }^{2}$ Department of Internal Medicine, Mohamed Tahar Maamouri Hospital, Tunisia

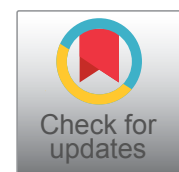

\begin{abstract}
Introduction: Primitive bone tumours of the pelvis represent a considerable challenge for carcinologic surgeons. Resections made in the acetabular zone could disrupt the continuity of the pelvic ring. The morbidity that comes after such a procedure requires a reconstruction surgery in order to improve the functional outcome. In this aim, multiple techniques have been reported.
\end{abstract}

Case report: We report the case of a 23 -year-old male with no medical history diagnosed with a right pelvic bone osteosarcoma located in Enneking zones I and II. He first underwent neoadjuvant chemotherapy followed by a large resection of the whole bone. A reconstruction was then made using a vascularized fibular autologous graft and stabilized by a long customized lombo-femoral plate. Recovery of walking was possible using crutches. No recurrence was note at one year follow-up.

Conclusion: Malignant primitive tumours of the pelvis threaten the vital and functional prognoses of patients. It is now possible to perform conservative resections in more than $80 \%$ of case without compromising the survival of patients or their functional outcome.

\section{Keywords}

Osteosarcoma, Pelvis, Reconstruction, Plate

\section{Introduction}

Ten to $15 \%$ of primitive malignant bone tumours are localized around the acetabulum [1,2]. Their prevalence is as much as 10 new case per year per million [3]. Regardless their histological variety, these tumours raise two major technical challenges : fist, large in resection margins have to be respected while resection of a tumour that is often of considerable size because of frequent diagnosis delay [4-7]; second, a complex reconstruction is often mandatory because of the disruption of the pelvic ring. In order to improve the functional outcome after large pelvic resections of these tumours, multiple techniques have been reported such as arthrodesis, prosthetic reconstructions and different bone grafting. We report a right pelvic bone reconstruction using a vascularized fibular autologous graft and stabilized by a customized lombo-femoral plate.

\section{Case Report}

A 23-year-old otherwise healthy male presented with unbearable pain of the right inguinal region, evolving for many weeks. Plain antero-posterior pelvis radiographs showed a Lodwick type II large lytic lesion of the right ilium with sur- rounding soft tissue calcifications (Figure 1). ComputedTomography scan (CT scan) confirmed this aggressive lesion of the rightilium (Figure 2). Magnetic Resonance Imaging (MRI) precised that the lesion was located in Enneking zones I and II. However, this first MRI was not able to assess the integrity of the coxo-femoral and sacro-iliac joints with confidence (Figure 3). The spreading investigations were all negative. A surgical biopsy of the lesion was made and the diagnosis of osteosarcoma was histologically confirmed. The patient underwent neoadjuvant chemotherapy. A second MRI assessment was then made, showing a mild regression of the tumour with no extension to joints. A large surgical resection of the whole

*Corresponding author: Zeineb Alaya, Department of Internal Medicine, Mohamed Tahar Maamouri Hospital, 8000 Nabeul, Tunisia

Accepted: November 10, 2020

Published online: November 12, 2020

Citation: Khalifa MA, Alaya Z, Hassini L, et al. (2020) Pelvic Osteosarcoma: Resection and Reconstruction Using A Customised Plate. J Orthop Surg Tech 3(2):199-204 
Citation: Khalifa MA, Alaya Z, Hassini L, et al. (2020) Pelvic Osteosarcoma: Resection and Reconstruction Using A Customised Plate. J Orthop Surg Tech 3(2):199-204

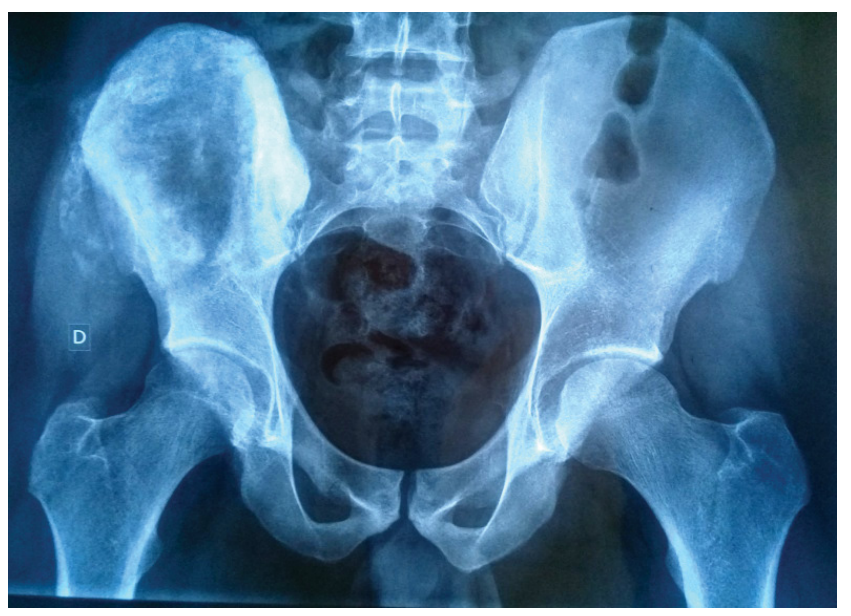

Figure 1: Plain antero-posterior pelvis radiograph showing a Lodwick type II large lytic lesion of the right ilium with surrounding soft tissue calcifications.

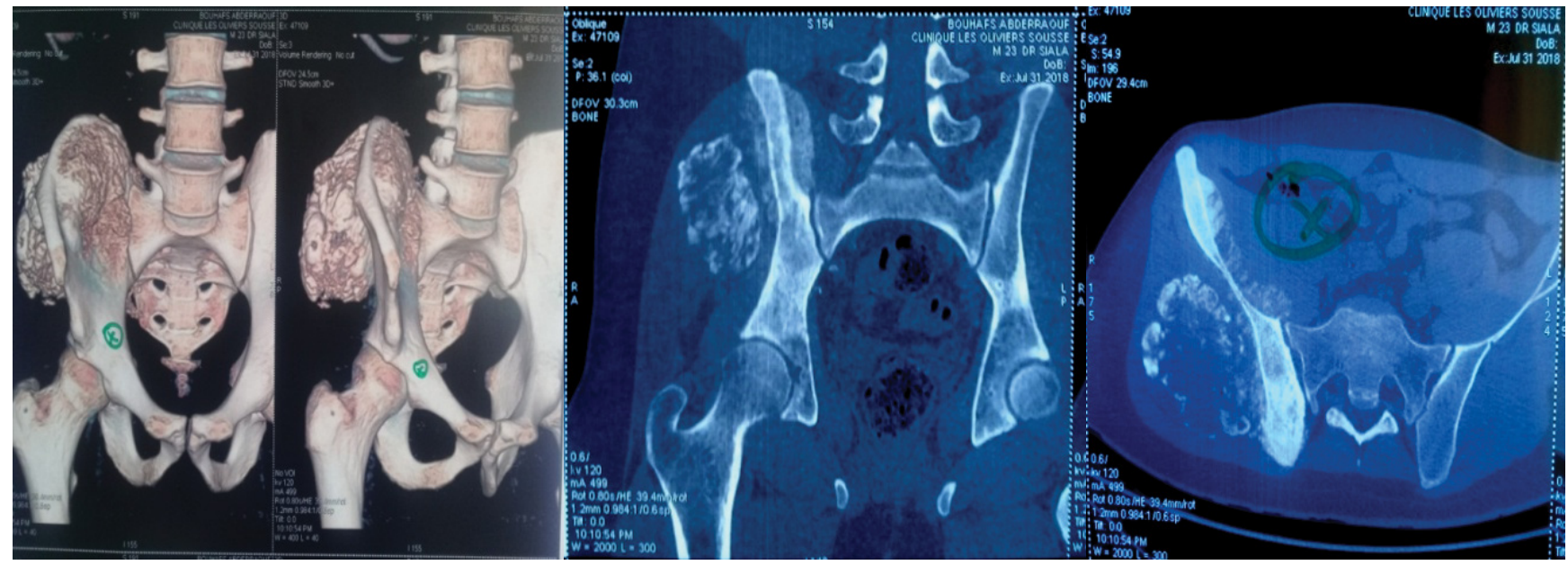

Figure 2: CT scan slices showing an aggressive lesion of the right ilium.

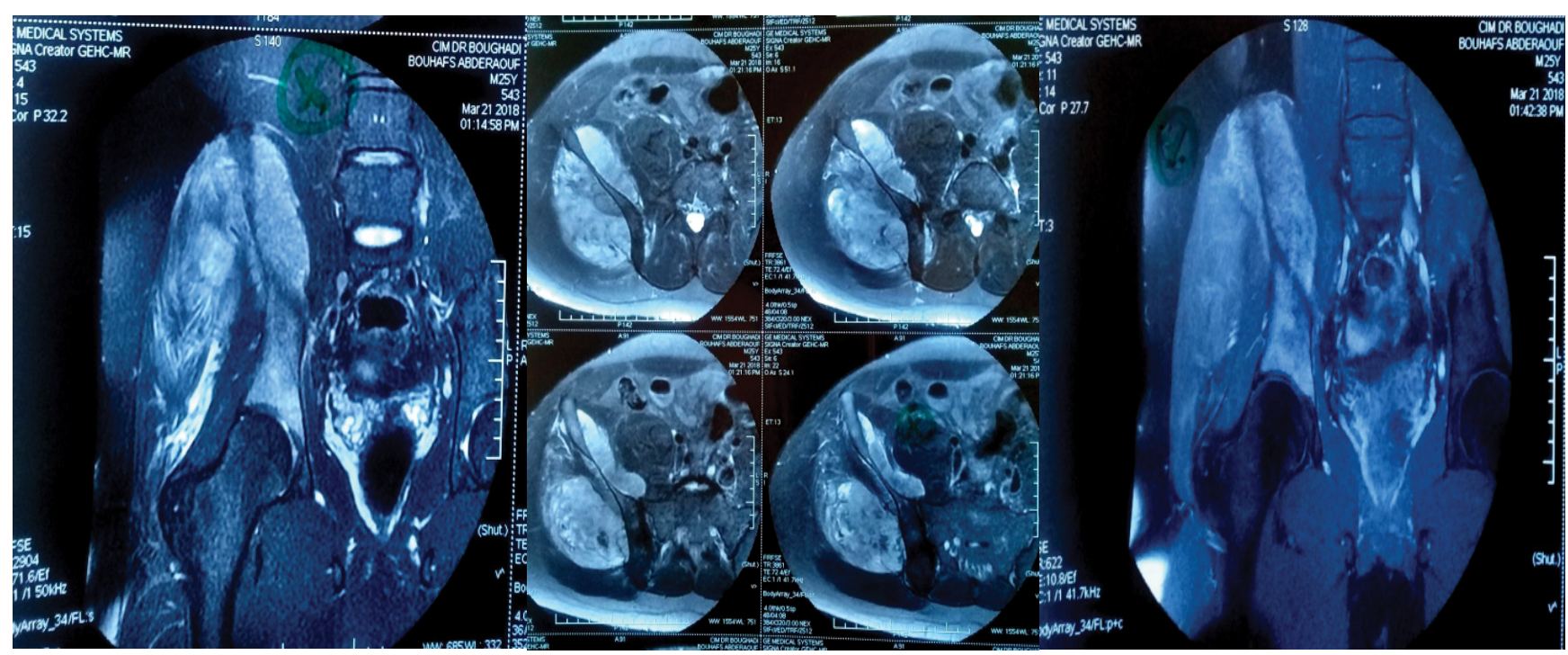

Figure 3: Pre-treatment MRI.

right pelvic bone was then performed. The reconstruction was made using a vascularised fibular graft and stabilized by a customized long lombo-femoral (Figure 4 and Figure 5). The post-surgery was simple and the wound healed at con- 
Citation: Khalifa MA, Alaya Z, Hassini L, et al. (2020) Pelvic Osteosarcoma: Resection and Reconstruction Using A Customised Plate. J Orthop Surg Tech 3(2):199-204

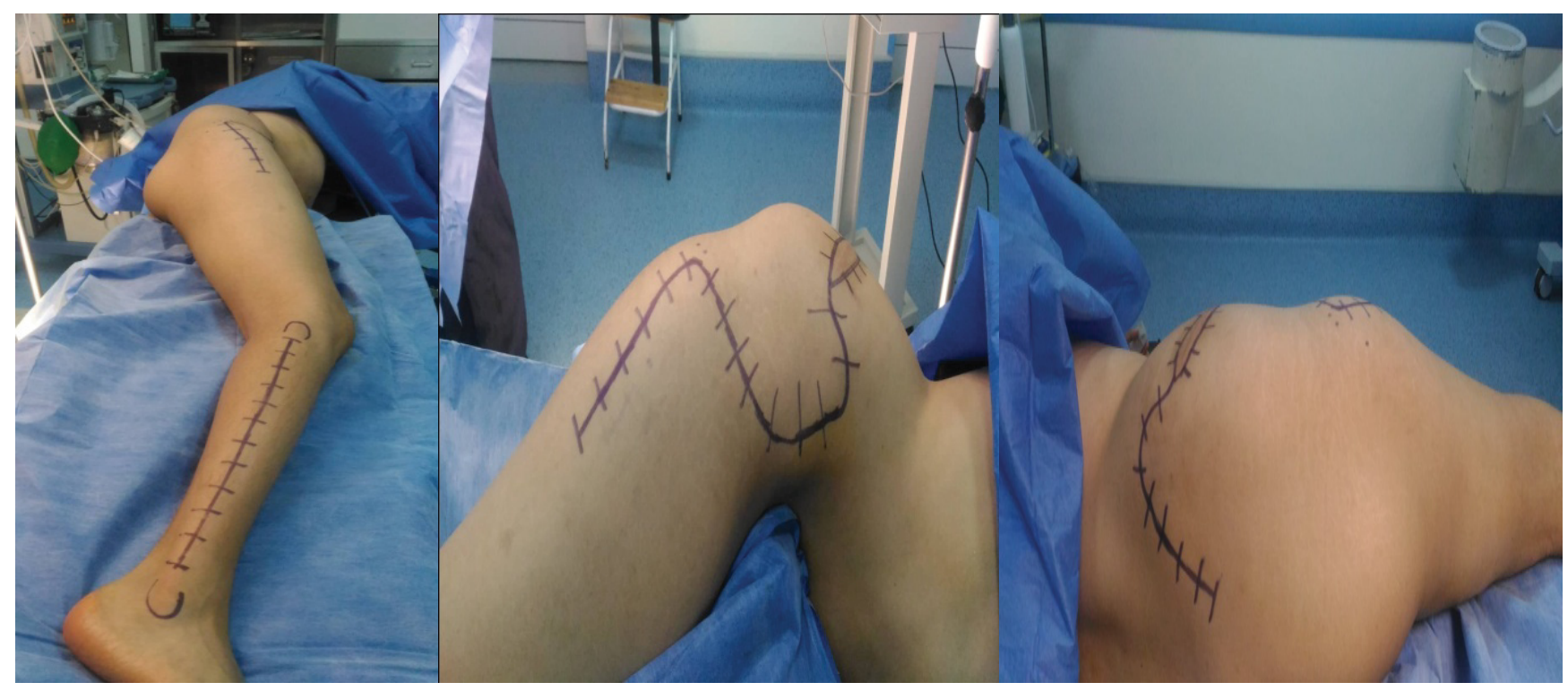

Figure 4: Installation and landmarks of the approach.

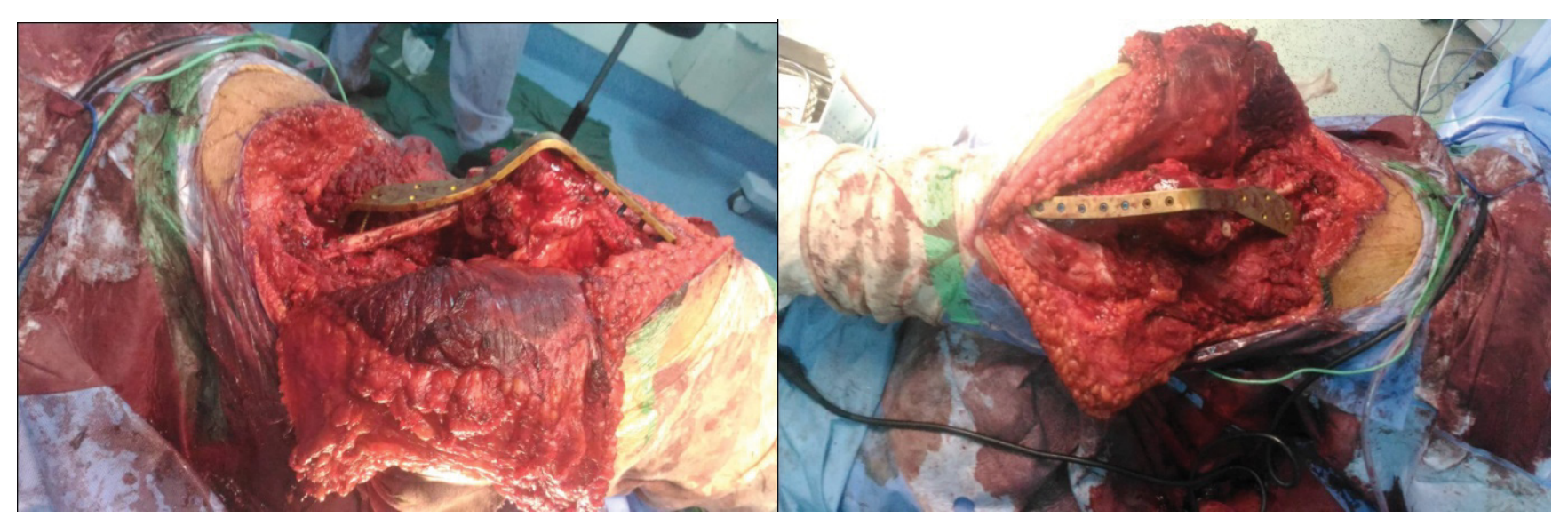

Figure 5: Peroperative views of monobloc resection andnonvascularized fibula reconstruction with custom plate osteosynthesis.

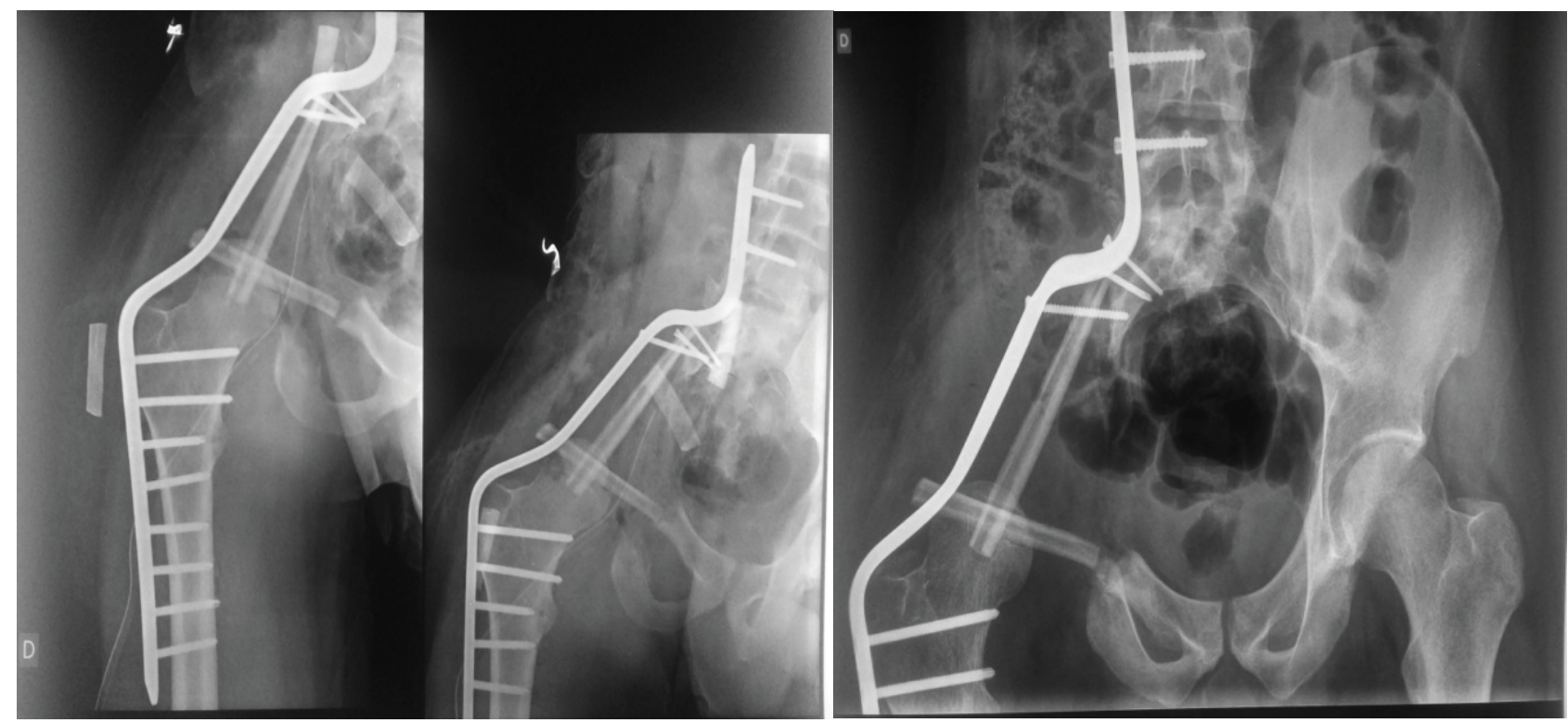

Figure 6: Postoperative X- RAY. 
ventional delay (Figure 6). Figure 7 shows the post-operative plain radiograph (Figure 7). A pelvi-crural cast was crafted and maintained for 6-weeks. The radiological control at 1-year follow-up showed an ongoing bone graft healing process (Figure 8). Functional outcome was average with the patient being able to walk using 2 crutches (Figure 9).

\section{Discussion}

A large number of musculoskeletal tumors can affect the pelvis. Several studies have reported bone sarcomas as the most common lesions, followed by soft tissue sarcomas and metastatic lesions. The most frequently observed pelvic sarcoma is chondrosarcoma, followed by osteosarcoma [8]. Ten to $15 \%$ of all primary bone tumors are located in the pelvic bone of which chondrosarcoma in adults, Ewing's sarcoma in children, and osteosarcoma in adolescents represent the most common histological subtypes [9] Pelvic resections are challenging and complex. They are technically difficult due to sometimes the size of the tumor is very large, and anatomically it closes to pelvic viscera and neurovascular structure.Previously, hemipelvectomy was the standard surgical treatment for primary pelvic sarcomas. Recently, with advancements in surgical techniques and chemotherapy and ra- diation therapy, limb salvage procedures have also emerged as viable modalities. Di Caprio, et al. [10] compared in their meta-analysis conservative procedures to amputation. They found that the decision making was mainly based on the patient's age, the size and the localization of the tumour. Another study of the Italian and the Scandinavian Sarcoma Groups found that high doses neoadjuvant chemotherapy allow a conservative surgical treatment in $90 \%$ of cases but increased liver and renal toxicity [11]. Progressively, pelvic amputation became a rare indication. Furthermore, life quality and function scores were significantly poorer with amputation compared to conservative procedures [12,13], with similar results regarding tumour resection. Davis, et al. [14] reported a series of 36 patients among whom 12 underwent amputation. Shortform-36 (SF-36) score was lower in this group compared to patients who underwent conservative procedures (45 versus $71.1, p=0.03$ ).

Though limb salvage surgery remains difficult and challenging, various methods were used to reconstruct the hemipelvis after periacetabular reconstruction resection, including ischiofemoral arthrodesis or pseudarthrosis, iliofemoral arthrodesis or pseudarthrosis [15], massive allograft [16,17], autoclaved autograft [18], allograft prosthetic composite [19],

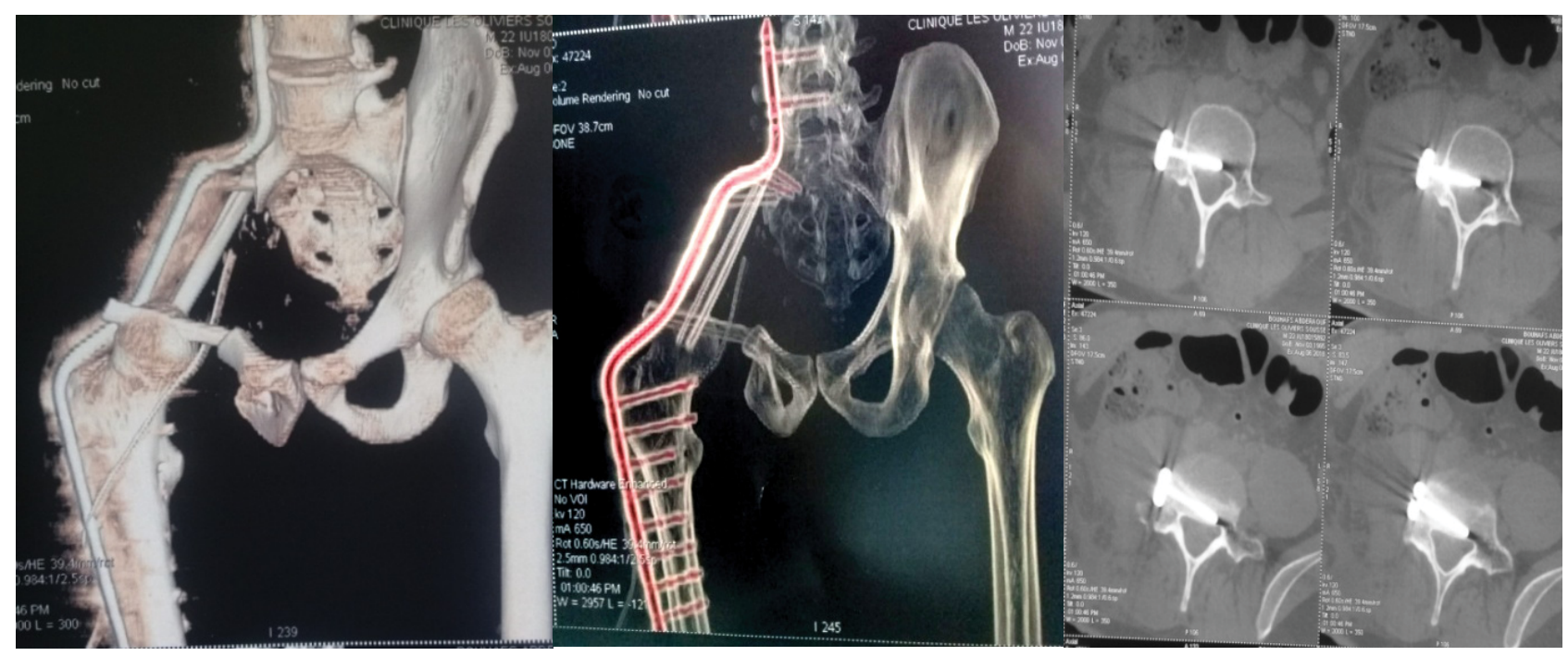

Figure 7: Postoperative CT scan.

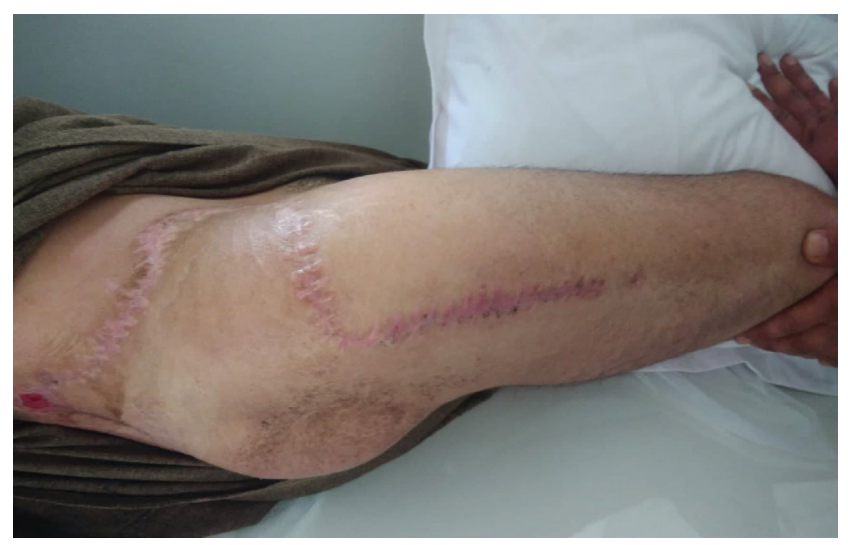

Figure 8: Operation scar.

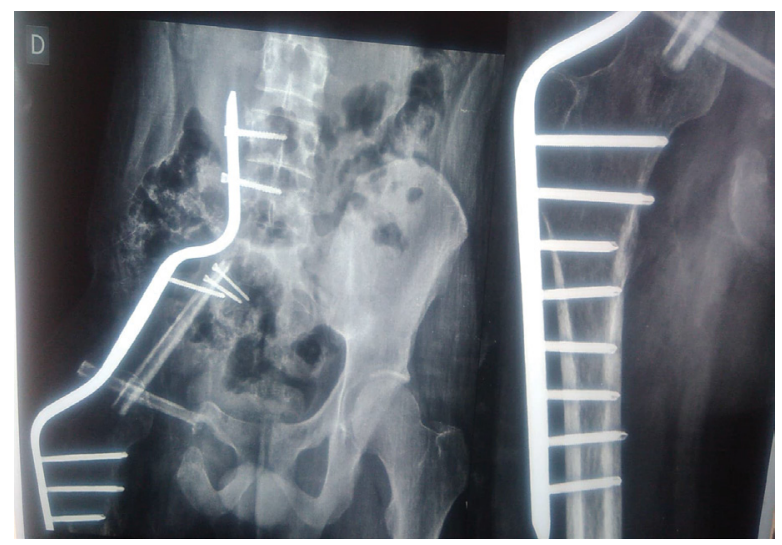

Figure 9: Plain radiograph at 2 year follow-up. 
custom-made endoprosthesis combined with hip arthroplasty [20], modular saddle prosthesis [21] or modular hemipelvic endoprosthesis. Each reconstruction modality has its own risks and benefits [22].

As reported in the early series of Ennekingand, et al. [23], O Connor, et al. [24], Capanna, et al. [25] and Campanacci, et al. [6], patients requiring wide muscle resection with nerve sacrifice underwent arthrodesis.llio-femoral, ischio-femoral, femoro-obturator and femoro-sacral arthrodesis have been used. The choice depended on the resection zone in the classification of Enneking and Dunham. In the present case, the patient underwent a lombo-femoral arthrodesis. We did not use a saddle or stemmed prostheses for reconstruction because those were very expensivein our country.

The principle of arthrodesis after pelvis resection is to achieve solid union between proximal femur and the remaining pelvis (iliofemoral, ischiofemoral, or sacrofemoral) using plate, screw, or wire. In the present case, we used a customized plate fixes proximally in the $4^{\text {th }}$ and $5^{\text {th }}$ lombar vertebral body and in the femur distally.

The treatment of this pathology is associated to a high rate of complications and a significant declining of quality of life regardless the surgical technique. A high rate of revision is observed in these patients [26]. The two most common post-operative complications are wound infection (with an incidence of 10 to $47 \%$ ) and local recurrence of the tumour (with an incidence of 28 to 35\%) [20,27]. Han, et al. Showed that surgical margin was the factor most closely related to the local recurrence [28]. Our case showed no local recurrence until last follow up. It was probably due to we can achieve wide margin during surgery.

Functional results depend on the extent of resection and the method of reconstruction [29]. The disadvantage of arthrodesis includes loss of the hip joint function. Fusion rates of femoropelvic arthrodesis are $<50 \%$, in most patient, a stable and painless pseudoarthrosis develops, but with a comparable functional result to that of the alternative reconstruction options [30]. Higher non-union rates were reported with patients undergoing chemotherapy and/or radiation therapy $[6,15]$. Until last follow up, functional result of our patient was average with disappearance of pain and resumption of walking using 2 crutches.

\section{Conclusion}

Primitive pelvic bone tumours represent a challenging pathology for surgeons. Acetabular resections disturb the continuity of the pelvic ring and require a reconstruction. In order to improve functional outcome after this delabrating surgery, multiple techniques have been reported. Current data shows that complication rate is high and quality of life is significantly compromised regardless the reconstruction procedure used. Since complication in surgery of pelvis tumor is quite high, approach in the management of pelvis tumor needs multidiciplinary team.

\section{References}

1. Apffelstaedt JP, Driscoll DL, Spellman JE, et al. (1996) Complica- tions and outcome of external hemipelvectomy in the management of pelvic tumors. Ann Surg Oncol 3: 304-309.

2. Duman BB, Gunaldi M, Ercolak V, et al. (2012) Retrospective analysis of 498 primary soft tissue sarcomas in a single Turkish centre. Asian Pac J Cancer Prev 13: 4125-4128.

3. D Andon A, Vassal G, Oberlin O, et al. (2004) Les tumeurs osseuses primitives. Institut Gustave-Roussy. Villejuif.

4. Fuchs B, Hoekzema N, Larson DR, et al. (2009) Osteosarcoma of the pelvis: Outcome analysis of surgical treatment. Clin Orthop 467: 510-518.

5. Sluga M, Windhager R, Lang S, et al. (2001) The role of surgery and resection margins in the treatment of Ewing's sarcoma. Clin Orthop 392: 394-349.

6. Campanacci M, Capanna R (1991) Pelvic resections: The rizzoli institute experience. Orthop Clin North Am 22: 65-86.

7. Hillmann A, Hoffmann C, Gosheger G, et al. (2003) Tumors of the pelvis: Complications after reconstruction. Arch Orthop Trauma Surg 123: 340-344.

8. JG Garcia, A Martinez, RJ Filho, et al. (2018) Epidemiological characteristics of patients with pelvic tumors submitted to surgical treatment. Rev Bras Ortop 53: 33-37.

9. SE Puchner, PT Funovics, C Bohler, et al. (2017) Oncological and surgical outcome after treatment of pelvic sarcomas. PLoS One 12: e0172203.

10. Di Caprio MR, Friedlaender GE (2003) Malignant bone tumors: Limb sparing versus amputation. JAm Acad Orthop Surg 11: 2537.

11. FerrariS, SmelandS, MercuriM, et al. (2005) Neoadjuvant chemotherapy with high-dose Ifosfamide, high-dosemethotrexate, cisplatin, and doxorubicin for patients with localized osteosarcoma of the extremity: A joint study by the italian and scandinavian sarcoma groups. Jclin Oncol Off J Am Soc Clin Oncol 23: 88458852.

12. Hopyan S, Tan JW, Graham HK, et al. (2006) Function and upright time following limb salvage, amputation, and rotationplasty for pediatric sarcoma of bone. J Pediatr Orthop 26: 405-408.

13. Ginsberg JP, Rai SN, Carlson CA, et al. (2007) A comparative analysis of functional outcomes in adolescents and young adults with lower-extremity bone sarcoma. Pediatr Blood Cancer 49: 964969.

14. Davis AM, Devlin M, Griffin AM, et al. (1999) Functional outcome in amputation versus limb sparing of patients with lower extremity sarcoma: A matched case-control study. Arch Phys Med Rehabil 80: 615-618.

15. Fuchs B, O Connor MI, Kaufman KR, et al. (2002) Iliofemoral arthrodesis and pseudarthrosis: A long-term functional outcome evaluation. Clin Orthop 397: 29-35.

16. Bell RS, Davis AM, Wunder JS, et al. (1997) Allograft reconstruction of the acetabulum after resection of grade II B sarcoma. Intermediateterm results. J Bone Joint Surg 79A: 1663-1674.

17. Matejovsky Z Jr, Matejovsky Z, Kofranek I (2006) Massive allografts in tumour surgery. Int Orthop 30: 478-483.

18. Harrington KD (1992) The use of hemipelvis allografts or autoclaved grafts for reconstruction after wide resection of malignant tumors of the pelvis. J Bone Joint Surg 74: 331-341.

19. Schwameis E, Dominkus M, Krepler P, et al. (2002) Reconstruc- 
tion of the pelvis after tumor resection in children and adolescents. Clin Orthop Relat Res 402: 220-235.

20. Abudu A, Grimer RJ, Cannon SR, et al. (1997) Reconstruction of the hemipelvis after excision of malignant tumors. Complications and functional outcome of prostheses. J Bone Joint Surg 79: 773-779.

21. Aljassir F, Beadel GP, Turcotte RE, et al. (2005) Outcome after pelvic sarcoma resection reconstructed with saddle prosthesis. Clin Orthop Relat Res 438: 36-41.

22. Y Kollender, S Shabat, J Bickels, et al. (2000) Internal hemipelvectomy for bone sarcomas in children and young adults: Surgical considerations. Eur J Surg Oncol 26: 398-404.

23. Enneking WF, Dunham WK (1978) Resection and reconstruction for primary neoplasms involving the innominate bone. J Bone Joint Surg Am 60: 731-746.

24. Connor MI, Sim FH (1989) Salvage of the limb in the treatment of malignant pelvic tumors. J Bone Joint Surg Am 71: 481-494.
25. Capanna R, Manfrini M, Pignatti G, et al. (1990) Hemipelvectomy in malignant neoplasms of the hip region. Ital J Orthop Traumatol 16: 425-437.

26. Hoffmann C, Gosheger G, Gebert C, et al. (2006) Functional results and quality of life after treatment of pelvic sarcomas involving the acetabulum. J Bone Joint Surg Am 88: 575-582.

27. Biau DJ, Thévenin F, Dumaine V, et al. (2009) Ipsilateral femoral autograft reconstruction after resection of a pelvic tumor. J Bone Joint Surg Am 91: 142-151.

28. Ilkyu Han, YM Lee, HS Cho, et al. (2010) Outcome after surgical treatment of pelvic sarcomas. Clin Orthop Surg 2: 160-166.

29. Wei G, Dasen L, Xiaodong T (2007) Reconstruction with modular hemipelvic prostheses for periacetabular tumor. Clin Orthop Relat Res 461: 180-188.

30. AF Mavrogenis, K Soultanis, P Patapis, et al. (2012) Pelvic resection. Orthopaedics 35: 232-243. 